\title{
Erratum: TinyDB2: Porting a Query Driven Data Extraction System to TinyOS2.x
}

\author{
Bhaskar Biswas and Himanshu Singh \\ Department of Computer Engineering, \\ Institute of Technology, Banaras Hindu University \\ Varanasi, India \\ \{bhaskar.cse, himanshu.singh.cse07\} @itbhu.ac.in \\ http://itbhu.ac.in/cse/index.php/people/faculty/28.html
}

D.C. Wyld et al. (Eds.): NeCoM/WeST/WiMoN 2011, CCIS 197, pp. 298-306, 2011.

(C) Springer-Verlag Berlin Heidelberg 2011

DOI 10.1007/978-3-642-22543-7_75

Due to a serious case of plagiarism this paper has been retracted. 